Oil of lavender

Absolute alcohol

Iso-propyl alcohol

Pyridine

Amyl alcohol
Light yellow ppt. changing to black

Yellow solution, white ppt.

Orange solution, white ppt.

Light yellow solution, white ppt.

Brown solution, white ppt.

\title{
Summary
}

This article deals with the preparation of germanium tetra-iodide, its analysis, the determination of its melting point, crystal form and specific gravity, its color at various temperatures, the sublimation and dissociation of the compound, and some of its chemical properties.

ITHACA, NEW YORK

[Contribution from the BIOphysical, Laboratories of the Cancer Commission of HARVARD UNIVERSITY]

\section{THE POTENTIAL DIFFERENCE BETWEEN GLASS AND ELECTROLYTES IN CONTACT WITH THE GLASS}

\author{
By WALTER S. HUGHES ${ }^{1}$ \\ Received September 14, 1922
}

Haber and Klemensiewicz ${ }^{2}$ found that the potential difference between electrolytes in contact with the 2 sides of a thin glass wall is determined in part by the hydrogen-ion concentration of the electrolytes. Haber's apparatus was arranged as in Fig. 1.

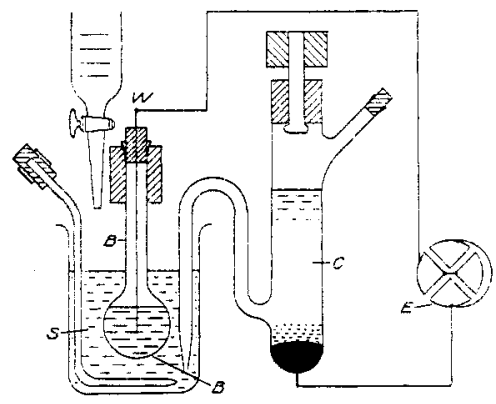

Fig. 1.

$S$ is the solution whose composition is to be varied; $B$ is a glass bulb with walls $0.06 \mathrm{~mm}$. thick, filled with potassium chloride solution; $W$ is a platinum wire dipping into the potassium chloride solution; $\mathrm{E}$ is a quadrant electrometer; $\mathrm{C}$ is a mercury-calomel-potassium-chloride electrode.

The potential difference between the quadrants of the electrometer was measured by observing the deflection of the electrometer mirror. This potential difference is the algebraic sum of several potential differences at different points in the system, of which the following are the most important: (1) that between the interior of the glass composing the bulb and the solution (S) outside the bulb; (2) that between the solution $S$ and the potassium chloride of the calomel electrode. When the composition of the solution $S$ is changed, these potential differences are obviously the only ones in the system which vary.

The change in the diffusion potential at the potassium chloride liquid junction is known to be small (about 10-20 millivolts) under the condi-

${ }_{1}^{1}$ Research Fellow, Cancer Commission of Harvard University.

2 Klemensiewicz, Z. Physik. Chem., 67, 385-431 (1909). 
tions of Haber's experiments. The potential difference between the interior of the glass forming the bulb and the solution outside varies, according to Haber, in a linear manner with the logarithm of the hydrogen-ion concentration of the solution $\mathrm{S}$. He suggests that the variations in surface potential of glass might be made the basis of an electrometric titration method and shows several titration curves in which he plots electrical potentials as a function of the cubic centimeters of acid or alkali added to the solution $\mathrm{S}$. He does not give graphs showing the relationship between glass surface potential and hydrogen-ion concentration as measured by the hydrogen electrode.

The purpose of the present investigation, therefore, was to make a further study of the potentials measured by Haber, in order to answer the following questions: (1) What is the exact character of the curve

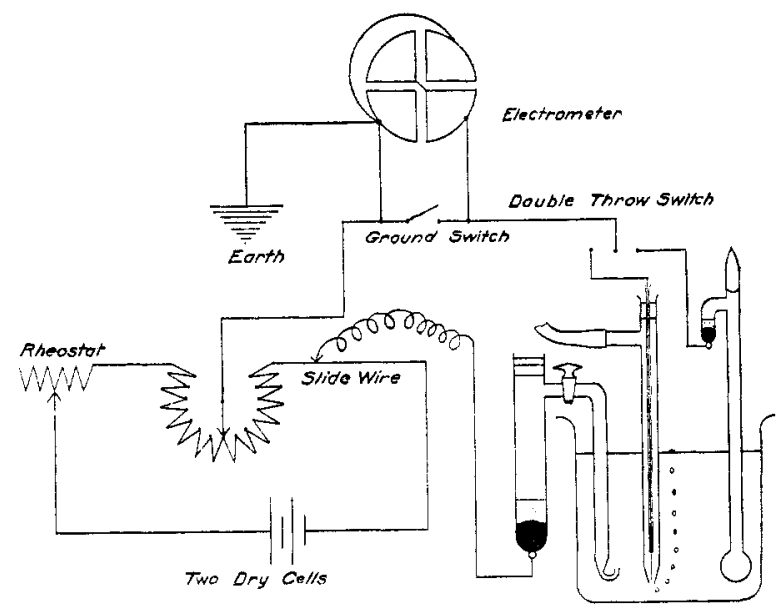

Fig. 2.

representing the relationship between glass surface potential and hydrogenion concentration as measured by the hydrogen electrode? (2) Can the potential of a glass surface be used as a measure of hydrogen-ion concentration in cases where the hydrogen electrode cannot be used, as in the presence of strong oxidizing agents? (3) What are the conditions under which the glass-surface potential is not a reliable measure of hydrogen-ion concentration, and what other ions influence this potential? Further work is being done on this phase of the problem.

In a preliminary study of glass-surface potentials, the set-up of our apparatus was at first essentially that used by Haber. Later we modified our apparatus and used the quadrant electrometer as a null instrument in connection with a potentiometer as shown in Fig. 2. Instead of making connections with the electrolyte inside the bulb by means of a platinum wire dipping into the electrolyte, we used a mercury-calomel-hydrochloric- 
acid electrode contained in a side neck on the stem of the bulb. Inside the bulb was $34 \%$ hydrochloric acid.

This type of bulb connection afforded an electrode potential of the mercury-calomel-hydrochloric-acid electrode in the side neck which was reproducible and constant. Furthermore as the hydrochloric acid inside of the bulb is always more acid than the electrolyte outside the bulb, the potential of the acid inside is, therefore, always negative with respect to the potential of the outside solution. When a neutral electrolyte is used inside the bulb, the potential inside may be positive or negative with respect to the potential outside, thus necessitating a reversing switch in the potentiometer connections.

The tubing used in making the bulbs was ordinary soft soda-lime glass. The bulbs were blown, filled without touching them and left in $N$ hydrochloric acid until a constant surface potential was reached before using. Between experiments the bulbs were left in distilled water or dil hydro. chloric acid.

A quadrant electrometer was used as a null instrument instead of employing the deflection of the electrometer as a direct measure of potential differences, as this arrangement eliminated the necessity of calibrating the electrometer deflections in terms of potential difference. Furthermore, the electrometer could be adjusted to a much higher degree of sensitivity and the measurements could thus be made more accurately.

The Use of a Quadrant Electrometer.-The deflection of a quadrant electrometer is a true measure of the potential difference between two parts of a circuit only when the resistance of the circuit between these parts is of a lower order of magnitude than the resistance of the air gap between the electrometer vane and the ungrounded quadrants. If these quadrants are left completely isolated, a charge will leak from the vane (which may be 100 volts above or below earth potential) to the ungrounded quadrants. These quadrants may thus become charged to several tenths of a volt above or below the earth potential.

All connections in the circuit in which the electrometer is connected must be bright, clean metallic contacts. If this precaution is not observed. contact potentials at dirty connections may amount to several hundredths of a volt.

Establishment of Glass-surface Potentials. - The potential difference between the electrolytes on each side of the glass bulb is established and maintained by leakage of the electricity through the bulb, the resistance of which is about 23 to 100 megohms. This is shown by the fact that when a charge is imparted to the ungrounded quadrant of the electrometer by touching it with a charged glass rod or bit of vulcanite, the charge leaks off almost immediately and the electrometer vane returns to its initial position. This leakage must take place through the thin glass of the bulb. 
and not along the surface of the stem, since the surface resistance of the glass was too large to measure, and must have been at least a hundred times the resistance of the bulb, which was easily measurable.

In order to determine the magnitude of the influence of surface leakage along the stem of the bulb on the potentials observed, we wound a platinum. wire around the neck of the bulb below the electrometer connection and then fused the wire into the surface of the glass. This "guard ring" was then earthed. Whether the guard ring was earthed or kept several volts above or below earth potential made no difference in the potentiometer readings. We also tried to measure the current forced along the surface of the neck of the glass bulb by connecting one pole of a 100-volt battery with the guard ring and the other pole with the solution in which the bulb dipped, the bulb being empty. No current could be detected with a galvanometer sensitive to $10^{-8}$ amperes.

The air-gap leakage from the charged electrometer vane to the ungrounded quadrants does not introduce an error of over 3 or 4 millivolts into the potentiometer reading. This may be shown by reversing the sign of the charge on the electrometer vane and checking the former reading of the potentiometer. The fact that the potentiometer null

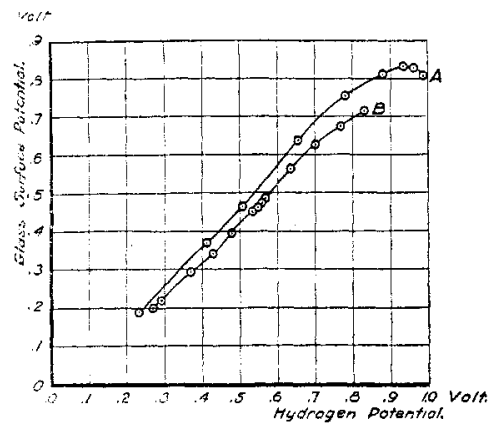

Fig. 3.

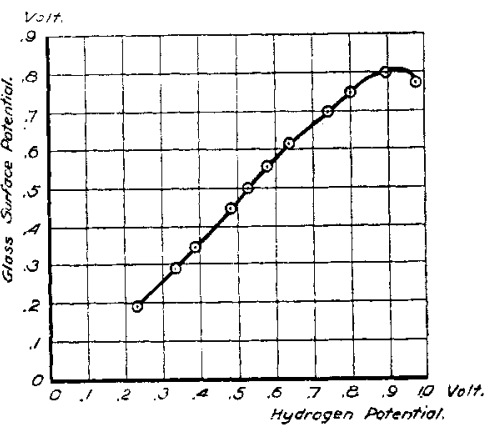

Fig. 4.

method can be used at all to measure the potential difference between the 2 sides of the bulb shows that the leakage through which the potential difference is established must be the largest leakage in the system. On account of the facts discussed above, many of Haber's precautions to avoid surface leakages are not very important, particularly the manner in which he suspended by silk threads his beaker containing the solutions. In damp weather, however, it is advisable to paraffin the surface of the bulb stem where the clamp holds it.

\section{Experimental Data}

The experimental results are presented in Fig. 3. The temperature was $25^{\circ}$ and the glass bulb was filled with $34 \%$ hydrochloric acid.

The solutions outside contained hydrochloric acid, acetic acid, sodium 
borate and caustic soda in various dilutions (mostly 0.1 to $0.01 \mathrm{~N}$ ) and were adjusted to give the required potential readings. The mercury-calomel electrode was filled with saturated potassium chloride solution. Since the glass-surface potentials were measured against the same calomel electrode as the hydrogen-electrode potentials, any variations in the liquidjunction potential where the calomel electrode dips in the solution would affect both the glass potential measurements and the hydrogen potential measurements in the same way.

The potential difference between the outside and inside of the bulb passes through a maximum as the hydrogen-ion concentration outside decreases from $10^{-12}$ to $10^{-13}(\pi=0.94-1.00$ v. $)$. The glass-surface potential is not a linear function of the logarithm of the hydrogen-ion concentration except when the hydrogen-ion concentration is greater than $10^{-11}$. Even then the linear relationship is only approximate.

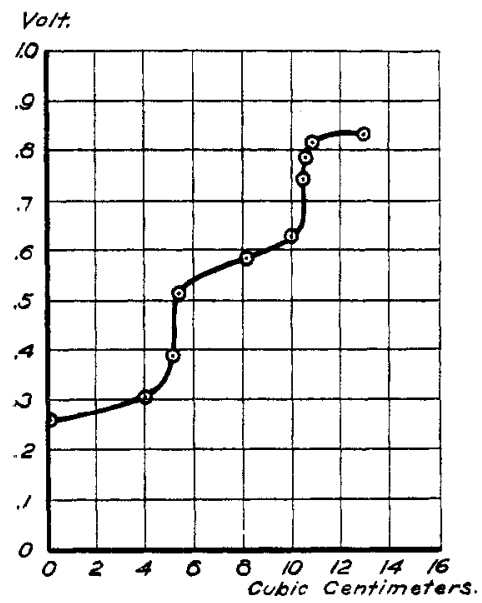

Fig. 5.

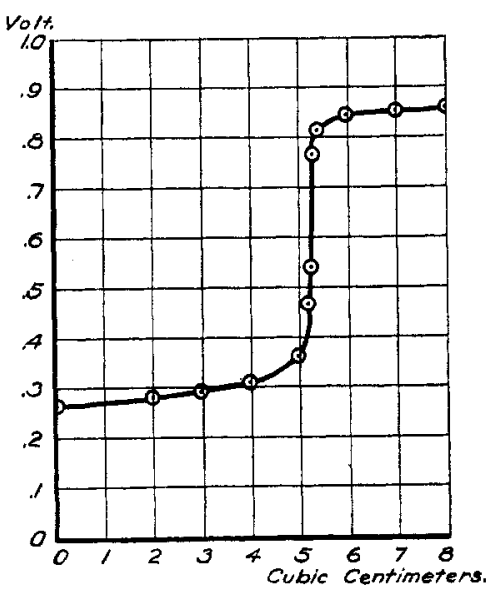

Fig. 6.

After the glass bulb had stood over-night in the alkaline solution last tabulated (having a hydrogen-ion concentration of about $1.8 \times 10^{-13}$ ) the potential difference between the sides of the bulb dropped from 0.807 to 0.776 ; at the same time the hydrogen-electrode potential dropped from 0.991 to 0.979 . This change in hydrogen potential was probably due to absorption of carbon dioxide from the air by the solution. The larger drop in glass-surface potential was due to the fact that a glass-surface potential against an alkaline solution changes continuously from the time the glass is first placed in contact with the solution, until an equilibrium value is approached after several hours. The potential of a glass surface is thus dependent on the length of time it has been in contact with an alkaline solution, as well as on the composition of the solution.

Fig. 4 shows how the glass-surface potential varies with the hydrogen potential as the hydrogen-ion concentration of the solution is increased. 
The electrometric method of titration suggested by Haber based on variations of glass surface potential may be used to titrate such acids as chromic and permanganic acids. Figs. 5 and 6 show how two such titrations were performed. In the former, $50 \mathrm{cc}$. of approximately 0.1 molal chromic acid was titrated with normal sodium hydroxide solution. The dissociation constants of the 2 hydrogen atoms in chromic acid are sufficiently different to cause two rapid rises in potential from small additions of alkali. In the latter, $50 \mathrm{cc}$. of approximately $0.105 \mathrm{~N}$ permanganic acid was titrated with $N$ sodium hydroxide solution.

This method could undoubtedly be used to titrate nitric, perchloric, iodic and other similar acids whose presence would render the use of the hydrogen electrode impossible. Some interesting data might be obtained concerning the degree of dissociation, number of replaceable hydrogen atoms, etc., of such acids. The method might also be useful in the presence of other substances which "poison" a hydrogen electrode.

Fig. 7 shows how glass-surface potential varies with hydrogen potential in a saturated potassium chloride solution containing various quantities

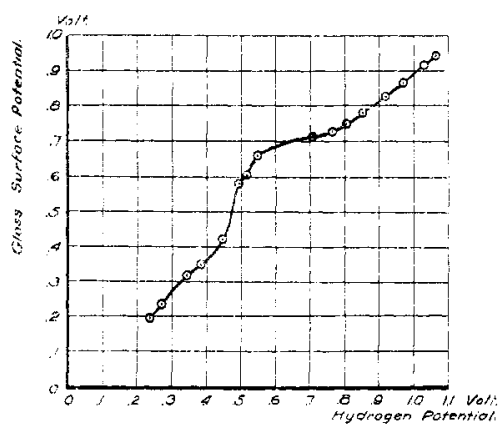

Fig. 7 .

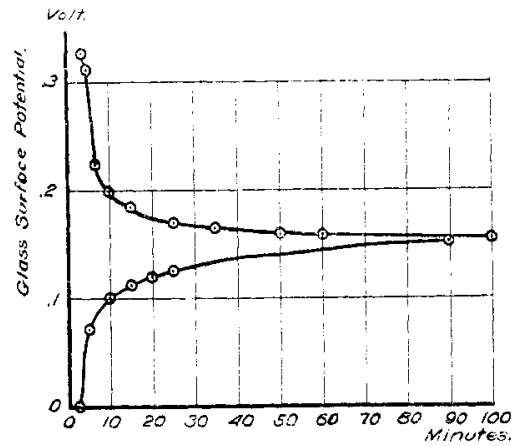

Fig. 8.

of hydrochloric acid and potassium hydroxide. The character of the curve is quite different from those of Figs. 3 and 4 . Sufficient data to explain these differences have not yet been obtained.

Curve B of Fig. 3 shows how glass-surface potential varies with the hydrogen-electrode potential in a $1 \%$ gelatin solution, to which was added various amounts of hydrochloric acid and sodium hydroxide. The temperature here was $26^{\circ}$.

These values show a nearly linear relationship between the two potentials, but the glass-surface potential is lower for a given hydrogen potential in the presence of gelatin than it would be in a dilute solution of acids, bases or salts (free from organic matter) giving the same hydrogen potential.

Before any acid or alkali was added to the gelatin solution the glass surface potential was 0.531 and the hydrogen potential 0.560 . These values 
do not fall anywhere on Curve B of Fig. 3. Probably the presence of electrolytes has some influence on the adsorption of gelatin on the glass surface.

\section{TABLE I}

\begin{tabular}{cccc}
\multicolumn{4}{c}{ Potentials IN ONE PER CENT. Gelatin Sol,utions } \\
$\begin{array}{c}\text { Glass-surface } \\
\text { potential }\end{array}$ & $\begin{array}{c}\text { Hydrogen-electrode } \\
\text { potential }\end{array}$ & $\begin{array}{c}\text { Glass-surface } \\
\text { potential }\end{array}$ & $\begin{array}{c}\text { Hydrogen-electrode } \\
\text { potential }\end{array}$ \\
0.200 & 0.270 & 0.461 & 0.556 \\
0.219 & 0.293 & 0.474 & 0.557 \\
0.291 & 0.371 & 0.486 & 0.567 \\
0.328 & 0.408 & 0.561 & 0.637 \\
0.340 & 0.431 & 0.625 & 0.702 \\
0.395 & 0.482 & 0.659 & 0.773 \\
0.451 & 0.540 & 0.714 & 0.834
\end{tabular}

Polarizing of Glass Surface.-When a current of about $10^{-6}$ amperes is forced through the wall of the bulb by applying a sufficient potential difference between the electrolytes inside and outside the bulb, the surfaces of the bulb become altered and the surface potentials after such treatment are changed. This unstable polarized condition of the glass surfaces takes about an hour to disappear, after the polarizing current has been stopped.

Table II and Fig. 8 show the recovery of normal potential on a glass surface after it has been polarized by forcing a current through it.

\begin{tabular}{|c|c|c|c|}
\hline \multicolumn{4}{|c|}{ TABLE II } \\
\hline \multicolumn{2}{|c|}{$\begin{array}{l}\text { Polarization voltage } 84 \text { volts, } \\
\text { inside }+ \text {, outside - }\end{array}$} & \multicolumn{2}{|c|}{$\begin{array}{l}\text { Polarization voltage } 84 \text { volts, } \\
\text { inside - outside }+\end{array}$} \\
\hline $\begin{array}{l}\text { Time after } \\
\text { polarization discont. } \\
\text { Min. }\end{array}$ & $\begin{array}{l}\text { Voltage out } \\
\text { - voltage in }\end{array}$ & $\begin{array}{l}\text { Time after } \\
\text { polarization discont. } \\
\text { Min. }\end{array}$ & $\begin{array}{l}\text { Voltage out } \\
\text { - voltage in }\end{array}$ \\
\hline 2.5 & 0.00 & 5 & +0.312 \\
\hline 5.0 & +0.072 & 10 & +0.200 \\
\hline 10 & +0.100 & 25 & +0.170 \\
\hline 20 & +0.119 & 50 & +0.160 \\
\hline 80 & +0.152 & 60 & +0.159 \\
\hline & & 120 & +0.153 \\
\hline
\end{tabular}

This effect is not due to the slow leakage of a charge from the ungrounded quadrant of the electrometer, for if a charge is imparted to the ungrounded quadrant by touching it with charged vulcanite, the charge leaks off immediately, as already mentioned.

The suggestion was made that the potential of a glass surface might be influenced by the presence of other ions than hydrogen ions, even in dilute solutions, particularly by the presence of polyvalent ions such as those of calcium, the sulfate radical, thorium $\left(\mathrm{Th}^{++++}\right)$, etc.

Many experiments were performed to test this suggestion. It was found that all dilute solutions $(0.1$ to $0.01 \mathrm{~N})$ adjusted to give the same hydrogen potential, gave the same glass-surface potential, regardless of what ions other than hydrogen were present. In some of these solutions the color 
of certain indicators was not a reliable measure of the hydrogen-ion concentration, as shown by the hydrogen electrode.

\title{
Summary
}

1. Glass-surface potentials are established and maintained by the passage of electricity through the glass.

2. Glass-surface potential is a linear function of hydrogen-electrode potential only through a limited range of values.

3. Variations in glass-surface potential may be used as the basis of an electrometric titration method in the presence of oxidizing agents which render the hydrogen electrode useless. In such cases the method might give data which could not be obtained in any other way.

4. The presence of certain substances (strong salt solutions or gelatin solutions) other than hydrogen ions affects glass-surface potentials.

Boston 17, MassachUSETTS

[Contribution from the Research Laboratory of Appited Chemistry, Massachusettrs Institute of TechNology, No. 58]

\section{THE SOLUBILITY AND SPECIFIC RATES OF HYDROLYSIS OF MUSTARD GAS IN WATER ${ }^{1}$}

\author{
By Robert E. WiLson, E. W. Futiler aNd M. O. Schur \\ Received September 15, 1922
}

Introduction

The previous paper by the writers dealt chiefly with the accelerating effect of various alkaline colloidal solutions on the rate of hydrolysis of mustard gas. The tendency of the reactions to reverse in acid solutions was, therefore, merely mentioned in passing. This paper is devoted primarily to an analysis of the hydrolytic reactions of mustard gas in neutral and acid solutions, and presents data as to its solubility and the magnitude of the four reaction rate constants for the progress and reversal of each of the two stages of its hydrolysis.

It has been shown in the previous paper that the hydrolysis of mustard gas takes place only in the aqueous phase, and not at the interface; that the rate is constant in alkaline solutions but is retarded in acid; and that the hydrolysis eventually goes to completion in all solutions.

'Throughout the first paper it was taken for granted that the hydrolysis took place in two stages, rather than that both chlorine atoms always split off simultaneously. This former assumption is the only reasonable one in view of the fact that the 2 chlorine atoms are separated by 5 intermediate atoms. It is further verified by two other independent observations.

1 Published by permission of General Amos A. Fries, Chief of the Chemical Warfare Service. 'This work was aided by a grant from the C. M. Warren Fund of the American Academy of Arts and Sciences, the assistance of which is gratefully acknowledged. 